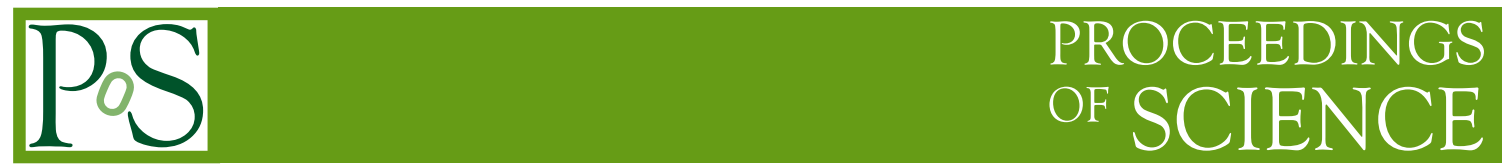

\title{
Orbital and radial excitations of static-light mesons
}

\section{Justin Foley}

Department of Physics, University of Swansea, Singleton Park, Swansea, SA2 8PP, UK.

\author{
Alan Ó Cais, Mike Peardon, and Sinéad M. Ryan* \\ School of Mathematics, Trinity College, Dublin 2, Ireland. \\ E-mail: ryan@maths.tcd.ie
}

Static-light meson spectroscopy is determined on a dynamical anisotropic lattice with all-to-all quark propagators. Results for the orbital and radial excitations are presented.

XXIVth International Symposium on Lattice Field Theory

July 23-28, 2006

Tucson, Arizona, USA

${ }^{*}$ Speaker. 


\section{Introduction}

The physics of particles with one or more heavy (bottom) quarks remains an interesting challenge for lattice QCD calculations. In particular, as the accuracy of some of the relevant experimental measurements reaches the percent level the onus is on theoretical (lattice) calculations to keep pace. Discretising the Dirac operator introduces mass-dependent errors which are large for the $b$ quark on currently accessible lattice spacings. HQET, NRQCD and the Fermilab formulation have been used for $b$ physics calculations as have relativistic simulations at lighter quark masses (typically charm) which are then extrapolated to the $b$ quark mass.

This work focuses on the spectrum of the static-light meson where the light quark is the strange quark. Experimentally, little is known of either the $B$ or $B_{s}$ mesons beyond the ground state. Theoretically, there are long-standing predictions from quark models including an inversion of orbitallyexcited multiplets [1,2]. Lattice calculations have a unique opportunity to make a first principles, model-independent determination of the spectrum, including the radial and orbital excitations.

The static lagrangian is the lowest order term in a $1 / m_{Q}$ expansion of the Heavy Quark Effective Theory (HQET). In this limit, where $m_{Q} \longrightarrow \infty$, approximate heavy-quark spin and flavour symmetries become exact. In principle, results from static simulations can have relevance for $b$ physics and can be used to elucidate properties of heavy hadron systems. However, in practice the rather poor signal-to-noise ratio in this theory has meant it is not widely used. This problem can be cured by a brute force increase in statistics or by using all-to-all propagators which increase statistics by considering source and sink operators on all (spatial) sites of the lattice. Previous work has shown the advantages of this approach $[3,4]$. In addition, improved signal-to-noise ratios have been found using a modified static action [5].

In this work a combination of all-to-all propagators on anisotropic lattices is used to determine precisely the spectrum of static-light mesons. The algorithm for simulating all-to-all propagators is described in detail in ref. [6]. We use a $3+1$ anisotropic lattice, where the temporal lattice spacing $a_{t}$ is fine while the spatial spacing, $a_{s}$ is coarse. For dynamical QCD simulations a simultaneous tuning of the quark and gluon anisotropies, $\xi_{q}$ and $\xi_{g}$ respectively, is needed and this is described in ref. [7].

\section{The static-light spectrum}

In the static theory the dynamics of the meson is governed by its light quark content with the heavy quark treated as a static colour source. In this limit hyperfine splitting disappears and we can classify mesons according to the angular momentum and parity of the light degrees of freedom, labelled $J_{l}^{\pi_{l}}$. The orbital excitations, $S, P$ and $D$ wave states, are then classified as

$$
J_{l}^{\pi_{l}}=\underbrace{\frac{1^{-}}{2}}_{S} \underbrace{\frac{1^{+}}{2}, \frac{3^{+}}{2}}_{P} \underbrace{\frac{3^{-}}{2}, \frac{5^{-}}{2}}_{D}
$$

To extract the physics of interest one must construct lattice interpolating operators for the light degrees of freedom which transform according to the irreducible representations (irreps) of the symmetry group, $O_{h}$. The continuum quantum numbers of the states appearing in these irreps 
can be determined from the subduction of the continuum symmetry group to $O_{h}$. Table 1 shows the angular momentum content of the three double-valued irreps of the group of proper rotations allowed by the lattice. Including parity simply doubles the number of irreps, so that the irreps of $O_{h}$ are for example $G_{1 g}, G_{1 u}$ where the subscript $g / u$ denotes a representation which is even/odd under parity. From Table 1 we can deduce that to compute states with energies up to $L=2$ five irreps

\begin{tabular}{c|c|c}
\hline Lattice irrep & Dimension & Continuum irrep \\
\hline \hline$G_{1}$ & 2 & $\frac{1}{2}, \frac{7}{2}, \ldots$ \\
$G_{2}$ & 2 & $\frac{5}{2}, \frac{7}{2}, \ldots$ \\
$H$ & 4 & $\frac{3}{2}, \frac{5}{2}, \ldots$ \\
\hline
\end{tabular}

Table 1: The irreps of the lattice rotation group and the angular momentum of the lowest-lying continuum counterparts.

are required. It is interesting to note that the $\frac{3}{2}^{-} D$-wave appears only in the $H_{u}$ representation but that the $\frac{5}{2}^{-}$is split between the $G_{2 u}$ and the $H_{u}$ representations. We will return to this issue when discussing the results.

\section{Results}

The results presented here were determined on an $8^{3} \times 80$ lattice with an anisotropy of $\xi=6$. Table 2 lists further details of this simulation. We use all-to-all propagators with one hundred

\begin{tabular}{c|c}
\hline \hline Volume & $8^{3} \times 80$ \\
Configurations & 249 \\
$\beta$ & 1.5 \\
$a_{s}$ & $0.2 \mathrm{fm}$ \\
$a_{t} m_{\text {light }}, a_{t} m_{\text {sea }}$ & -0.057 \\
$m_{\pi} / m_{\rho}$ & 0.55 \\
\hline
\end{tabular}

Table 2: Simulation details. The light valence and sea quark masses are degenerate and correspond approximately to the strange quark mass. The lattice volume, $8^{3} \times 80$ is approximately $(1.6 \mathrm{fm})^{3}$.

eigenvectors and two sets of time and colour diluted noise vectors. Both single-link and planardiagonal displacements were used to construct the operators needed to study the $S, P$ and $D$ wave continuum states. For optimal signals we averaged over the rows of each lattice irrep and used time reversal symmetry to increase statistics. The light quark fields were Jacobi smeared (six levels for the $S$ wave irrep and five for the $P$ and $D$ wave irreps). The optimised correlation functions were then calculated using the variational method.

\subsection{Orbital excitations of the static-light meson}

Figure 1 is a compendium of our results for the ground states masses across the five lattice irreps corresponding to $S, P$ and $D$ wave states. The plot shows the effective mass of the five 
states studied (one $S$ wave, two $P$ waves and two $D$ waves). The effective masses were determined using a $\chi^{2}$ minimisation fit and 1000 bootstrap subsamples were used to determine the statistical errors. The quality of the signal is exceptionally good and clear plateaux can be seen for all states,

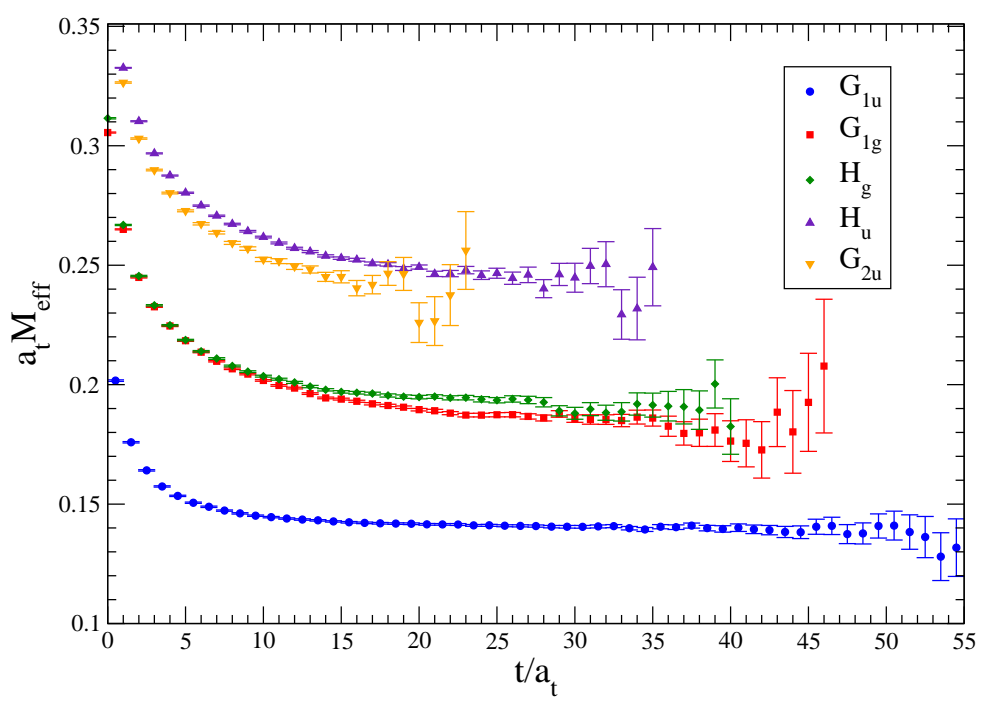

Figure 1: The effective masses of the fi ve states studied. The blue points are the $S$-wave, the red and green points are the two $P$ waves and the yellow and purple points are two $D$ waves. The plot shows the ground states only.

including the $D$ waves.

\subsection{Radial excitations of the static-light meson}

Using a large basis of operators and the variational method we have isolated what we believe to be radial excitations of the $S, P$ and $D$ wave states. Figure 2 shows the ground, first and second excited states of both the $S$ and $P$ wave mesons. Once again, clear plateaux are evident and energies, even for the second excited states, can be determined to a few percent precision. The same analysis techniques also yielded a good signal for the first excited state in the $H_{u}$ channel, although this state may not be a radial excitation. This is shown in Figure 3.

\subsection{Mass splittings}

In the static theory, the true physical observables are the mass splittings between states. We have investigated the precision with which these can be determined from the data described above. Figure 4 shows the mass differences between the ground state meson and its radial and orbital excitations. The data are plotted in lattice units and splittings are determined at the percent level including the splitting between the $\mathrm{S}$ wave and $\mathrm{D}$ wave states.

The plot shows no significant splitting between the ground states in the $G_{2 u}$ and $H_{u}$ representations which may imply it is the same (degenerate) $\frac{5}{2}^{-}$state. This could be interpreted as an inversion of the D-wave multiplet with the $\frac{5}{2}^{-}$state lighter than the $\frac{3}{2}^{-}$state. However, this has not been observed in previous lattice studies [3] and needs further investigation. In particular, although the statistical precision of these results is impressive the lattice volume is rather small for excited 

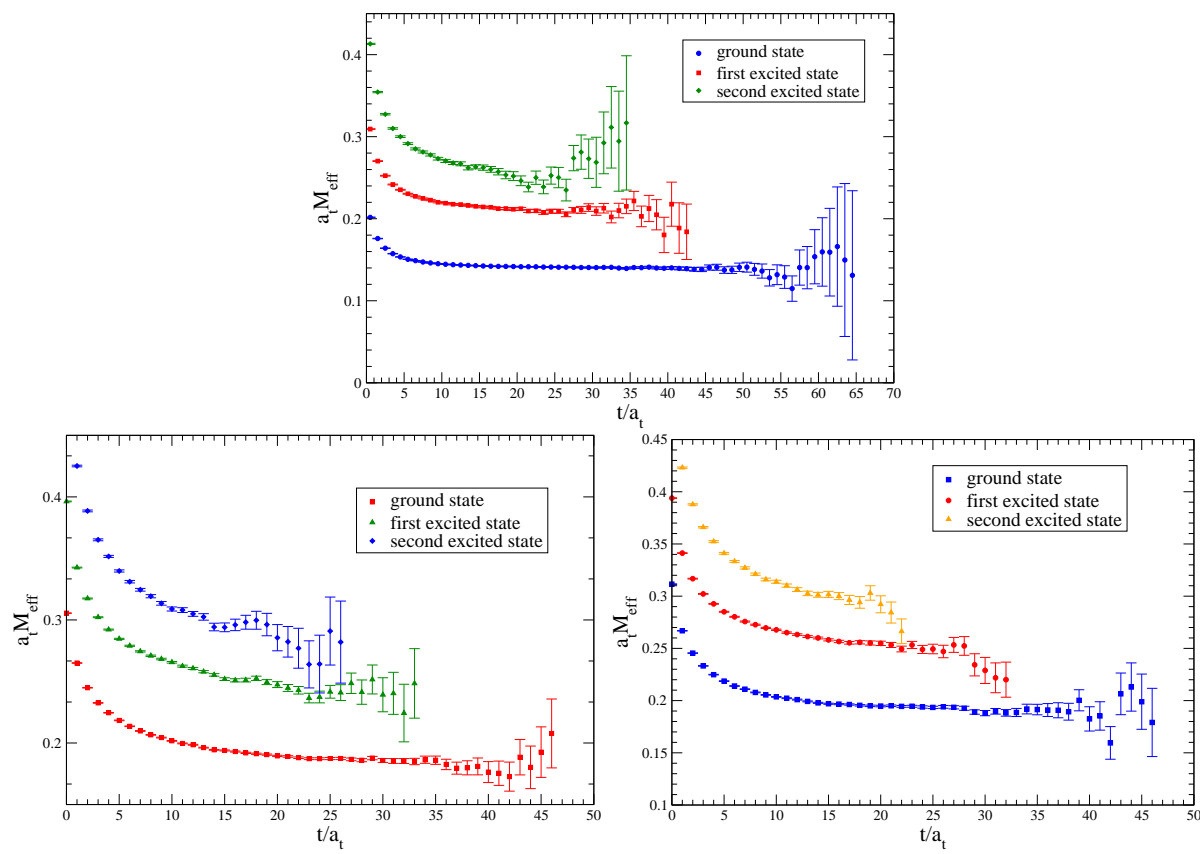

Figure 2: Effective mass plots showing the excitations in the $S$ wave (top plot) and $P$ wave irreps (the $G_{1 g}$ on the bottom left and the $H_{g}$ ) on the bottom right. The fi rst and second excited states are determined for all three mesons.

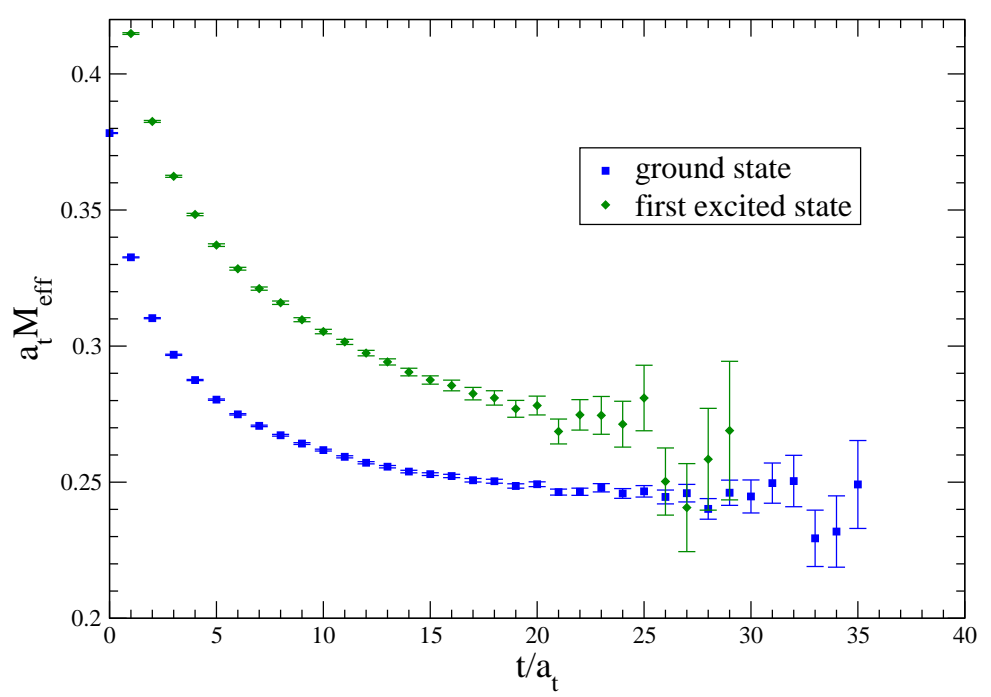

Figure 3: An effective mass plot showing the ground and fi rst excited state of the $H_{u}(D$ wave) irrep.

states. We are currently repeating this analysis on a larger lattice $(2.4 \mathrm{fm})^{3}$ to look for finite volume effects.

\section{Conclusions}

In this study we have presented results for the orbital and radial excitations of a static-light meson determined at the percent level of statistical precision. To achieve these results all-to-all 


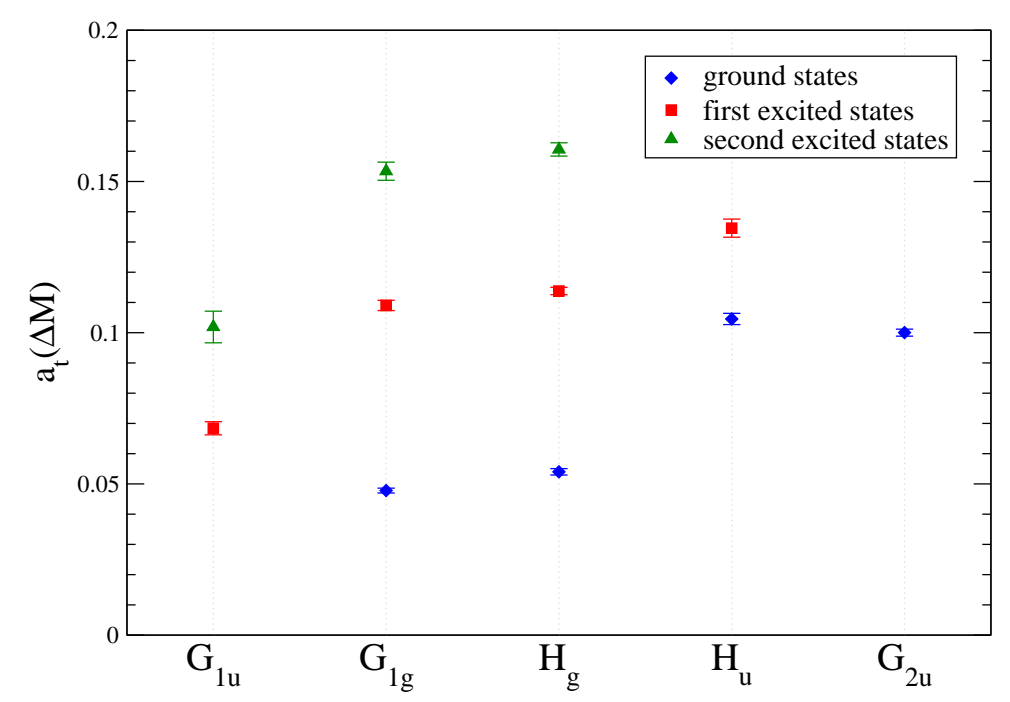

Figure 4: Mass differences, in units of $a_{t}$, between the $S$ wave and higher-lying states.

propagators were crucial: improving the signal-to-noise ratios and allowing for the easy constructing of a sophisticated basis of operators. This large variational basis played a key role, helping us to isolate the higher radial and orbital states of interest. Although a hundred eigenvectors were used in this work, separate studies have shown that approximately twenty are sufficient to reach the accuracy of the results presented here. Analysis is underway on a larger volume and with a larger variational basis and will be presented in a paper in the near future.

\section{References}

[1] H. J. Schnitzer, Phys. Lett. B226, 171 (1989).

[2] N. Isgur, Phys. Rev. D57, 4041 (1998).

[3] UKQCD, A. M. Green, J. Koponen, C. McNeile, C. Michael and G. Thompson, Phys. Rev. D69, 094505 (2004), [hep-lat/0312007].

[4] UKQCD, C. Michael and J. Peisa, Phys. Rev. D58, 034506 (1998), [hep-lat/9802015].

[5] M. Della Morte, A. Shindler and R. Sommer, JHEP 08, 051 (2005), [hep-lat/0506008].

[6] J. Foley et al., Comput. Phys. Commun. 172, 145 (2005), [hep-lat/0505023].

[7] R. Morrin, A. O. Cais, M. Peardon, S. M. Ryan and J.-I. Skullerud, Phys. Rev. D74, 014505 (2006), [hep-lat/0604021]. 